\title{
Variation Characteristics of Mass Concentration of Inhalable Particles in Qingdao, China
}

\author{
Zhixuan Wang ${ }^{1}$, Xiaomeng Shi ${ }^{2,3}{ }^{*}$, Yan Ma ${ }^{2,3}$, Xiaomin Wei ${ }^{1}$ \\ ${ }^{1}$ Physical Oceanography Laboratory, Ocean-Atmosphere Interaction and Climate Laboratory, Ocean University of China, \\ Qingdao, China \\ ${ }^{2}$ Qingdao Engineering Technology Research Center for Meteorological Disaster Prevention, Qingdao, China \\ ${ }^{3}$ Qingdao Meteorological Observatory, Qingdao Meteorological Bureau, Qingdao, China \\ Email: *shi198710@126.com
}

How to cite this paper: Wang, Z. X., Shi, X. M., Ma, Y., \& Wei, X. M. (2020). Variation Characteristics of Mass Concentration of Inhalable Particles in Qingdao, China. Journal of Geoscience and Environment Protection, 8, 192-201.

https://doi.org/10.4236/gep.2020.810014

Received: August 16, 2020

Accepted: October 26, 2020

Published: October 29, 2020

Copyright $\odot 2020$ by author(s) and Scientific Research Publishing Inc. This work is licensed under the Creative Commons Attribution International License (CC BY 4.0). http://creativecommons.org/licenses/by/4.0/

\begin{abstract}
The geographical condition of Qingdao, China is relatively special; the transport of various inland pollutants, the emissions of marine aerosol and local pollutants will have an impact on the changes of atmospheric aerosol concentration. By using the stability classification method, trajectory clustering analysis and the NOAA HYSPLIT model, the seasonal distribution characteristics of atmospheric inhalable particulate matter concentration in Qingdao, China and its relationship with meteorological conditions, mixed layer height, and the seasonal characteristics of Qingdao pollutant transport were analyzed. The results show that the variation trends of PM2.5 and PM10 were about the same, and there are obvious seasonal differences, which are high in winter and spring, and low in summer and autumn. The concentration of inhalable particulate matter has a negative correlation with temperature, wind speed and relative humidity. The concentration of inhalable particulate matter is distinct in different relative humidity ranges. When the wind speed is less than $3-4 \mathrm{~m} / \mathrm{s}$, there are more inhalable particles, while the mass concentration shows obvious reduction with the wind speed more than $4 \mathrm{~m} / \mathrm{s}$. There is a significant negative correlation between the mass concentration of pollutants and the daily maximum mixed layer height. The larger the concentration of pollutants, the smaller the thickness of the daily largest mixed layer. Conversely, the smaller the mass concentration of pollutants, the larger the thickness of the daily largest mixed layer. The pollutant transport in Qingdao has obvious seasonal characteristics. The air mass in spring, autumn and winter is mainly medium-long distance transport from Mongolia and southern Russia, and medium-short distance transport from Inner Mongolia and northeast of China. The source of air masses in summer is mainly transported from the eastern and sea areas.
\end{abstract}




\section{Keywords}

Variation Characteristics, Mass Concentration, Inhalable Particles, Qingdao

\section{Introduction}

Qingdao is located in the southern part of Shandong Peninsula, according to the Qingdao Environmental Status Bulletin during 2012-2015, although the number of days with good air quality in Qingdao is more than 260, the proportion of days with particulate matter as the primary pollutant is still high, whose ratio was as high as $97.2 \%$ in 2014. Han et al. (2014), Wan et al. (2015), and Song et al. (2016) pointed out that there was continuous pollution in Qingdao during the winter of 2013-2015, which was particularly severe in 2014. Among the pollutants in Qingdao, inhalable particulate matter of PM2.5 and PM10 accounts for a large proportion. Inhalable particulate matter causes the ventilating function of the alveoli and the function of the bronchioles to be destroyed by clogging localized tissue of the lung, which is harmful to human health.

The geographical condition of Qingdao is relatively special; the transport of various inland pollutants, the emissions of marine aerosol and local pollutants will have an impact on the changes of atmospheric aerosol concentration (Ke et al., 2014). In recent years, many experts and scholars researched air pollution in Qingdao, which mainly focuses on human health assessment ( $\mathrm{Hu}, 2003)$, pollution status investigation (Wang \& Gao, 2008) and prevention countermeasures research (Wang \& Guo, 1999; Li, 2006) and marine factors on the contribution of atmospheric aerosols (Xu \& Yao, 1997) and sedimentation flux (Chen et al., 2004); meanwhile, they also analyzed the relationship between inhalable particulate matter and meteorological elements (Ke et al., 2014; Huang et al., 2015; Kang et al., 2016).

The height of atmospheric mixed layer is one of the main factors affecting atmospheric diffusion, which plays an important role in air quality assessment and storage and distribution of pollutants ( $\mathrm{Li}$ et al., 2015). With the continuous development of research, scholars have found that the height of the mixed layer is closely related to the stability of atmosphere. In the field of environmental meteorology, the height of the mixed layer and the stability of atmosphere are commonly used in air pollution potential prediction and environmental assessment (Yuan et al., 2013).

Existing research mainly focuses on non-meteorological factors, such as human health assessment, pollution investigation, preventive measures, and marine factors, etc. Even if the research involves meteorological factors, it only analyzes the relationship between inhalable particulate matter and meteorological factors. Since the height of the mixed layer of the atmosphere is one of the main factors affecting atmospheric diffusion, this article not only analyzes the characteristics of atmospheric inhalable particulate matter in Qingdao, discusses the relationship between the concentration of particulate matter and meteoro- 
logical factors, but also analyzes the relationship between the height of particulate matter and the mixed layer, and the seasonal characteristics of pollutant migration in Qingdao.

\section{Data and Methods}

\subsection{Data}

Day-to-day surface observation data of Qingdao Meteorological Observatory from 2011 to 2015 (8 times a day, including meteorological elements such as total cloud cover, low cloud cover, wind direction and wind speed); Daily pollutant concentration data of PM10 (2011-2015) and PM2.5 (2013-2015) from Qingdao Shibei District North Atmosphere Monitoring Station; Calculating air mass trajectory used global reanalysis data $\left(2.5^{\circ} \times 2.5^{\circ}\right)$ provided by the National Oceanic and Atmospheric Administration (NOAA) in 2014.

Qingdao Shibei District North Atmosphere Monitoring Station is a representational station that can better characterize the air quality of Qingdao urban area, at the same time, the straight distance between the Shibei District North station and Qingdao Station is less than $1000 \mathrm{~m}$ so that the spatial difference can be neglected, and meteorological elements and pollution data can be considered the same place.

\subsection{Methods}

Pasquill (1961) proposed the stability classification method in 1961, and divided the solar radiation indicators into three categories to judge the atmospheric stability, which is strong, weak and weak. Turner (1964) added cloud height, cloud amount, and solar elevation angle to Pasquill's classification method to classify the atmospheric stability as the Pasquill-Turner (P-T) stability classification. The PT method classifies atmospheric stability into six levels: extremely unstable, unstable, weakly unstable, neutral, weakly stable and stable. It is replaced by A, B, C, D, E, F to express the six levels of atmospheric stability. In the China National GB/T13201-91 Standard issued by the National Environmental Protection Agency of China, the method of calculating the height of the mixed layer Lb (the national standard method) is defined based on the Joint Frequency Roche Method.

Trajectory clustering analysis is a multivariate statistical technique, which groups a large number of trajectories according to the similarity of trajectory space. In recent years, this analysis method has been applied in the study of air pollution more frequently (Brankov et al., 1998). In this paper, the NOAA-HYSPLIT model is used to track the backward trajectory of air masses of Qingdao in 2014, and the backward trajectory is classified by cluster analysis using TrajStat software (Wang et al., 2009).

\section{Results and Analysis}

\subsection{Inhalable Particle Change Curves}

Figure 1 is a daily average curve of PM10 and PM2.5 mass concentrations in 


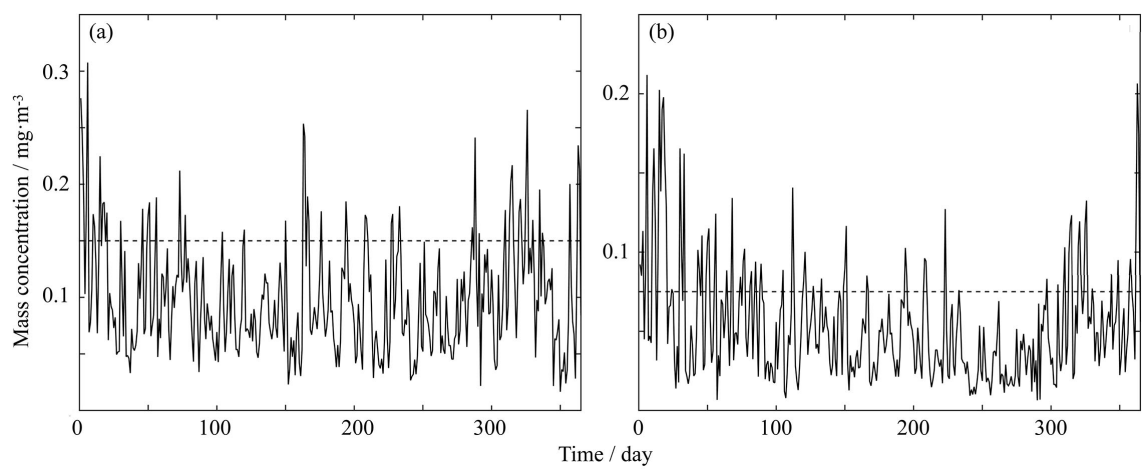

Figure 1. PM2.5 (a), PM10 (b) daily variation curve of mass concentration (solid line) and particulate matter limit standard line (dashed line).

2014. The variation trend of PM10 and PM2.5 mass concentration is about the same, and there are obvious seasonal differences, which are high in winter and spring, low in summer and autumn. Meanwhile, the PM10 mass concentration is slightly ahead of PM2.5. Through the lead (lag) correlation analysis, the highest correlation coefficient $(\mathrm{R}>0.9)$ is PM10 more ahead of PM2.5 than 3 days. Maybe the reason is that the coarse particles are more easily formed from the underlying surface, and the gravity sedimentation of the coarse particles themselves makes the coarse particles easier to remove than the fine particles.

\subsection{Relationship between Particle Concentration and Meteorological Elements}

Using daily average data, Huang et al. (2015) found that the concentration of pollutants in Qingdao was negatively correlated with cloud cover, precipitation and temperature, and was positively correlated with air pressure. Figure 2(a) also illustrates the negative correlation between inhalable particulate matter concentration and temperature, PM2.5 $(\mathrm{r}=-0.3)$ is more correlated with temperature than PM10 $(r=-0.12)$. In Figure 2(b) and Figure 2(c), there is also a negative correlation between inhalable particulate matter and wind speed and relative humidity, PM10 has a greater correlation with wind speed and relative humidity than PM2.5.

In addition, the author analyzed the relative humidity and wind speed by stages (Figure 3), and found that the correlation between relative humidity and particulate matter showed significant differences in different relative humidity intervals, as the relative humidity increased, the mass concentration of the pollutants is a single peak structure. The value of PM2.5 mass concentration greater than $0.075 \mathrm{mg} / \mathrm{m}^{3}$ occurs in the interval of relative humidity between $60 \%$ and $80 \%$, and the high concentration value of more than $0.15 \mathrm{mg} / \mathrm{m}^{3}$ is basically distributed in this interval. The probability of PM10 mass concentration greater than $0.15 \mathrm{mg} / \mathrm{m}^{3}$ appearing in the range of relative humidity between $60 \%$ and $80 \%$ is $54 \%$, but the high concentration value greater than $0.20 \mathrm{mg} / \mathrm{m}^{3}$ is mostly distributed in 40\% - 60\% (Figure 3(a), Figure 3(b), Table 1). As shown in Figures $3(\mathrm{c})-(\mathrm{d})$, when the wind speed is less than $3-4 \mathrm{~m} / \mathrm{s}$, the wind power is 
weak, the particles are difficult to have large-scale movement, and the inhalable particles are more concentrated in this wind speed interval. When the wind speed exceeds $4 \mathrm{~m} / \mathrm{s}$, the dynamic effect of the wind has a good dilution effect on the particulate matter, and the mass concentration is significantly reduced. However, the study also found that on a continuous high wind speed, the external force of the wind will cause the dust attached to the ground to be trapped in the atmosphere, causing the mass concentration of particulate matter to rise.

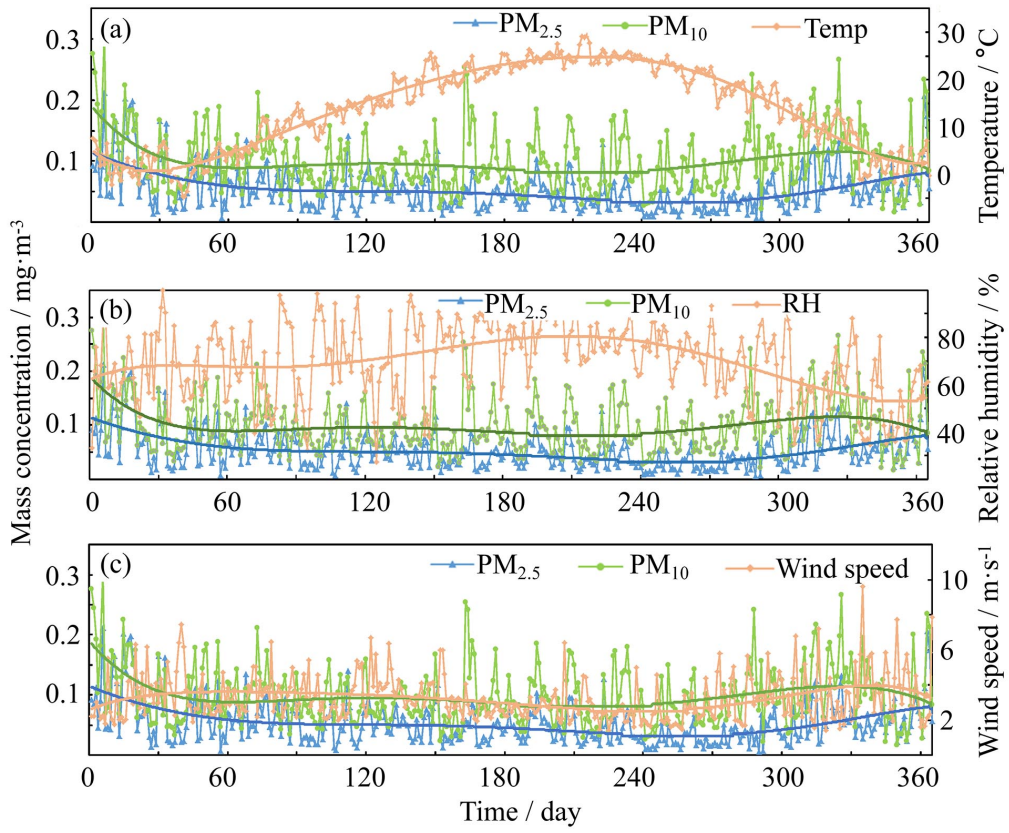

Figure 2. Daily change and fitting curve of PM10, PM2.5 mass concentration with temperature (a), relative humidity (b), wind speed (c).
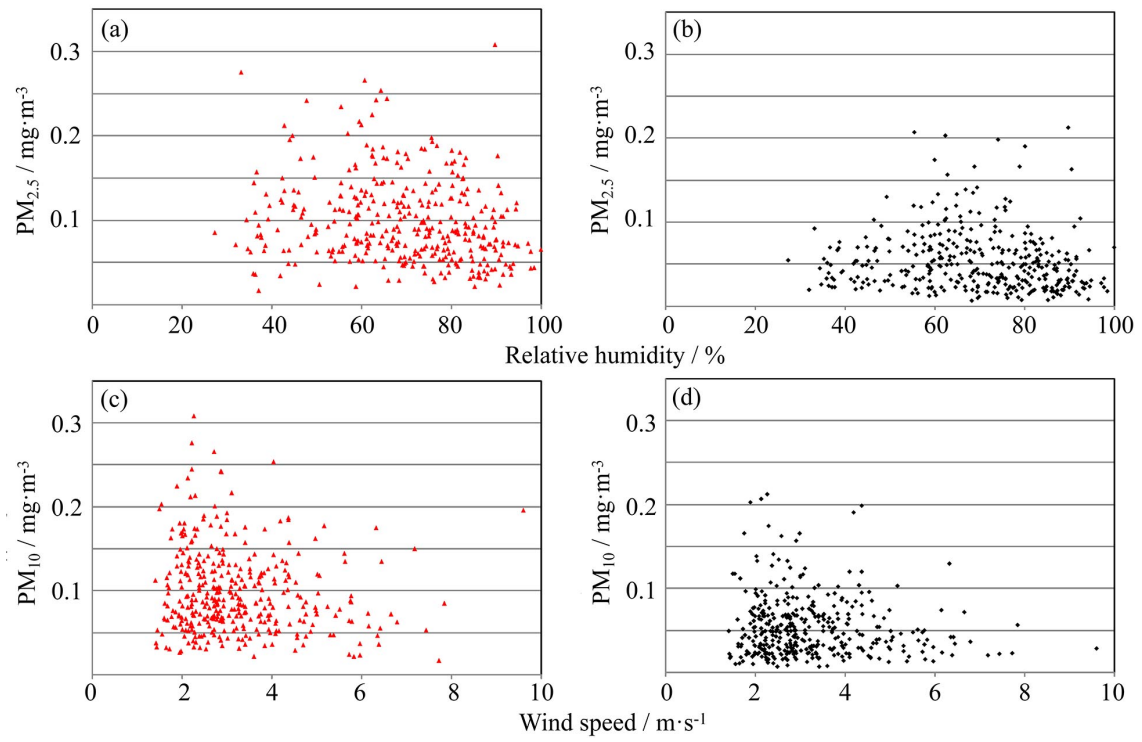

Figure 3. Scatter plot of PM10 and PM2.5 mass concentration with relative humidity (a) (b) and wind speed (c) (d). 
Table 1. Probability distribution table for excessive inhalable particles in different relative humidity ranges (2014).

\begin{tabular}{cccccc}
\hline $\begin{array}{c}\text { Relative Humidity } \\
\text { Mass Concentration } \\
\left(\mathrm{mg} / \mathrm{m}^{3}\right)\end{array}$ & $0 \%-20 \%$ & $20 \%-40 \%$ & $40 \%-60 \%$ & $60 \%-80 \%$ & $80 \%-100 \%$ \\
\hline $\begin{array}{c}\text { PM2.5 }>0.075 \\
(\text { Standard Line })\end{array}$ & $0 \%$ & $3 \%$ & $22 \%$ & $56 \%$ & $19 \%$ \\
$\begin{array}{c}\text { PM10 }>0.15 \\
(\text { Standard Line })\end{array}$ & $0 \%$ & $5 \%$ & $21 \%$ & $54 \%$ & $20 \%$ \\
PM2.5 $>0.15$ & $0 \%$ & $0 \%$ & $22 \%$ & $45 \%$ & $33 \%$ \\
PM10 > 0.20 & $0 \%$ & $11 \%$ & $56 \%$ & $33 \%$ & $0 \%$ \\
\hline
\end{tabular}

\subsection{Relationship between Particle Concentration and Height of Mixed Layer}

The mixed layer is a gas layer in which the turbulent atmosphere of the atmosphere is strongly mixed and the pollutants can be fully diluted and diffused, so the height change is closely related to the concentration of the pollutants. The thicker the mixed layer, the larger the area of attenuation and diffusion of the contaminants, and the lower the contaminant concentration. The maximum mixed layer thickness reflects the maximum atmospheric capacity that can be diluted, and the maximum range of dilution and diffusion of pollutants in the vertical direction, which is one of the indicators of pollution prediction (You et al., 2010). Through matching and correlation analyzing the daily maximum mixed layer height of Qingdao (Shi et al., 2016) and two types of inhalable particulate matter-PM10 (2011-2015), PM2.5 (2013-2015)-mass concentration changes (Figure 4), it can be seen that there is a significant negative correlation between the mass concentration of air pollutants and the maximum mixed layer height. After 9-point sliding average filtering data, the correlation coefficients of the maximum mixed layer height and PM10 and PM2.5 are -0.13 and -0.12 , passed the $t$ test of $99 \%$ significance. It showed that the greater the concentration of pollutants, the smaller the thickness of the largest daily mixed layer; on the contrary, the smaller the mass concentration of pollutants, the greater the thickness of the largest daily mixed layer.

\subsection{The NOAA-HYSPLIT Model of Inhalable Particulate Matter}

The NOAA-HYSPLIT model was used to track the trajectory of air mass trajectories of Qingdao in 2014, and the backward trajectory was classified by cluster analysis using TrajStat software. As shown in Figure 5(a), Figure 5(c) and Figure 5(d), the clustering trajectory of spring, autumn and winter is mainly northwestward, and a group of air masses is from the eastern sea in spring and autumn. The northwestward transport is mainly medium and long distance transport in Mongolia and south of Russia, and the northward and northeastward transport is mainly the medium and short distance particulate transport from China's Inner Mongolia and Northeast China. The main air mass sources in different seasons are different: in the spring, the medium and long distance 
transport in Mongolia and south of Russia accounted for the largest proportion at $44.6 \%$; in the autumn, the pollutants mainly transported by short-distance transport was from the northwest and the local area accounting for 39.6\%; in the winter, the medium and short distance transport from the northwestern border of China accounted for $49.4 \%$. As shown in Figure 5(b), summer air mass sources are mainly transported from the eastern and southern seas. It can be covered out from the above analysis that there are mainly three types of air masses in the urban area of Qingdao, which names the northwest sand source, the local source and the ocean source.

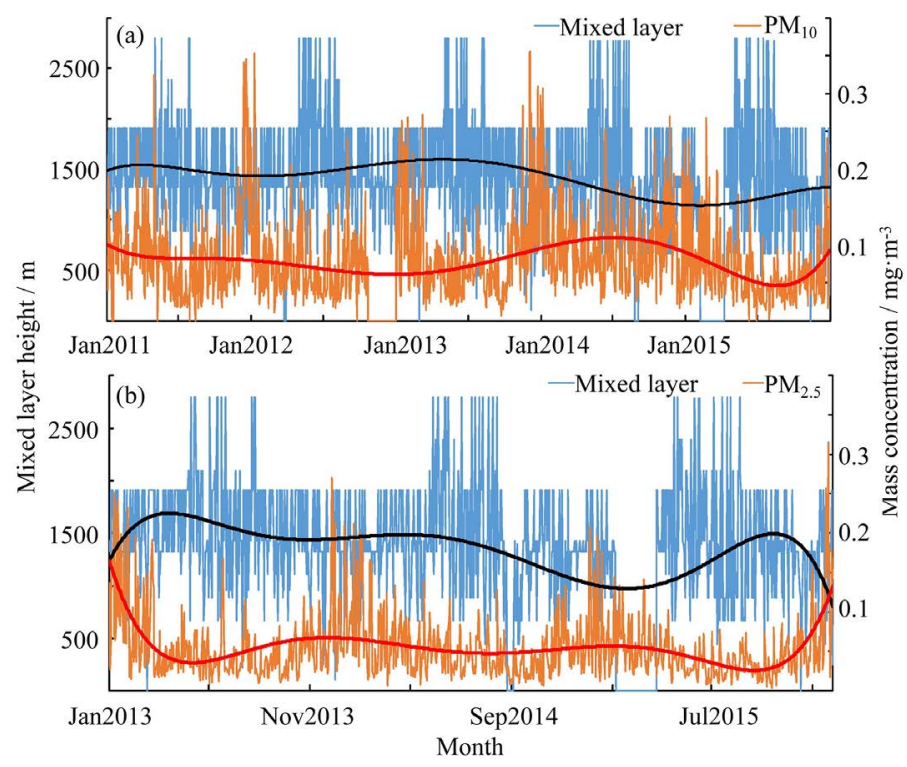

Figure 4. Change and fitting curve (black, red) of Qingdao daily maximum mixed layer height (blue) and PM10 ((a) 2011-2015), PM2.5 ((b) 2013-2015) mass concentration (orange).
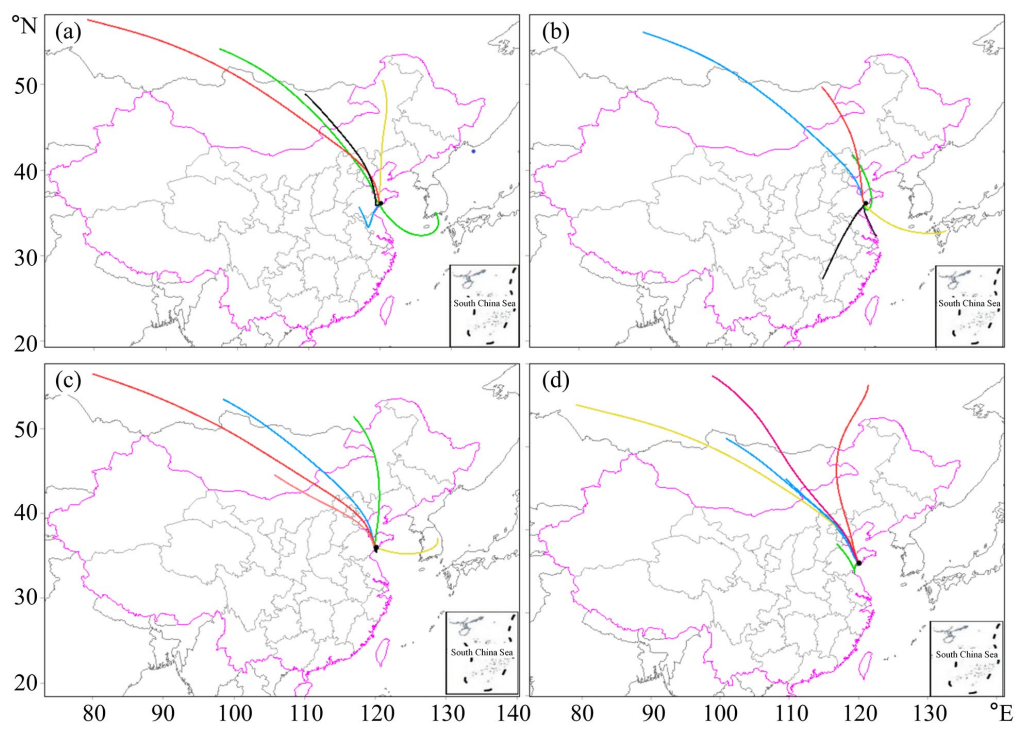

Figure 5. Seasonal cluster analysis of the air mass backward trajectory in 2014 . (a) Spring, (b) Summer, (c) Autumn, (d) Winter. 
$\mathrm{Xu}$ et al. (2017) used the HYSPLIT4 model to perform a cluster analysis on the air mass trajectory of the polluted weather in Qingdao, and found that the polluted weather caused by polluted air masses in Qingdao accounted for 64\%, and the cold air carried the polluted air masses in Beijing, Tianjin, Hebei and other places. The polluted weather caused by the southbound accounted for $36 \%$. Sun et al. (2015) comprehensively used meteorological observations, NOAA HYSPLIT backward trajectories, BC and Lidar to monitor the occurrence and development of a typical air pollution process in Qingdao in the late summer and early autumn (September 8) in 2014. Perform analysis. It is preliminarily judged that this pollution process is a relatively typical pollution process that is synergistically affected by local sources and external sources under adverse weather conditions. The local pollution source is mainly motor vehicle exhaust pollution. The above-mentioned literature also supports our findings and confirms that the analysis results of this study are repeatable.

\section{Conclusion and Discussion}

The trend of mass concentration of PM10 and PM2.5 in Qingdao is about the same, and there are obvious seasonal differences, which are high in winter and spring, low in summer and autumn.

The concentration of inhalable particulate matter in Qingdao has a negative correlation with temperature, wind speed and relative humidity. With the increase of relative humidity, the mass concentration of pollutants has a single peak structure. The high concentration of inhalable particulate matter appears in relative humidity at $60 \%-80 \%$. When the wind speed is less than $3-4 \mathrm{~m} / \mathrm{s}$, the wind power is weak, and the particles are difficult to have large-scale movement; when the wind speed exceeds $4 \mathrm{~m} / \mathrm{s}$, the mass concentration will be significantly reduced.

There is a significant negative correlation between the mass concentration of air pollutants in Qingdao and the maximum mixed layer height. The greater the mass concentration of pollutants, the smaller the thickness of the largest daily mixed layer; conversely, the smaller the mass concentration of pollutants, the greater the thickness of the largest daily mixed layer. The pollutant transport in Qingdao has obvious seasonal characteristics. In spring, autumn and winter, the northwest air mass transport is mainly medium and long distance transport in Mongolia and south of Russia, and the northward and northeast transport is mainly the medium and short distance transport of grain in China's Inner Mongolia and Northeast China; in summer, the air mass is mainly from the east and the southern seas.

\section{Acknowledgements}

This work was supported by project ZR2019PD002 supported by Shandong Provincial Natural Science Foundation and Qingdao Bureau Research Subject for Youth (2016qdqxq9). 


\section{Conflicts of Interest}

The authors declare no conflicts of interest regarding the publication of this paper.

\section{References}

Brankov, E., Rao, S. T., \& Porter, P. S. (1998). A Trajectory-Clustering-Correlation Methodology for Examining the Long-Range Transport of Air Pollutants. Atmospheric Environment, 32, 1525-1534. https://doi.org/10.1016/S1352-2310(97)00388-9

Chen, X. M., Feng, L. J., Zhang, H. A., Qi, J. H., \& Zhang, M. P. (2004). Deposition Fluxes of Several Metals in TSP in Qingdao Region. Marine Environmental Science, 22, 18-20.

Han, Y. Q., Liu, C., Wang, N., \& Meng, X. X. (2014). Weather Review of Shandong Province in Winter 2013 (December 2013-February 2014). Shandong Meteorology, 34, 77-79.

$\mathrm{Hu}$, Y. (2003). Evaluation on Effects of Qingdao Air Pollution On Economic Loss of Human Health. Chinese Journal of Public Health, 19, 940-941.

Huang, R., Guo, L. N., Ma, Y., \& Yu, S. B. (2015). Relationship between Air Quality and Meteorological Conditions from 2006 to 2012 in Qingdao. Journal of Meteorology and Environment, 31, 37-43.

Kang, G. H., Sun, X. C., Han, Y. Q., \& Song, J. J. (2016). Analysis of Spatial and Temporal Distribution Characteristics of Air Pollution in Shandong Province. Shandong Meteorology, 1, 13-17.

Ke, X. S., Sheng, L. F., Kong, J., Hao, Z. T., \& Qu, W. J. (2014). Variation of Atmospheric Particle Number Concentrations in Qingdao and Its Impact on Visibility. Environmental Science, 35, 15-21.

Li, H. Y. (2006). Study on Methods of Improving Air Quality in Qindao. Qingdao: Ocean of University of China.

Li, M., Tang, G. Q., Huang, J., Liu, Z. R., An, J. L., \& Wang, Y. S. (2015). Characteristics of Winter Atmospheric Mixing Layer Height in Beijing-TianjinHebei Region and Their Relationship with the Atmospheric Pollution. Environmental Science, 36, 1935-1943.

Pasquill, F. (1961). The Estimation of the Dispersion of Windborne Material. Australian Meteorological Magazine, 90, 33-49.

Shi, X. M., Wei, X. M., Bi, W., Ma, Y., Sun, J. L., \& Yi, L. (2016). Variation Characteristics of Mixed Layer Height in Qingdao and Its Relationship with Air Pollution. Shandong Meteorology, 36, 1-6.

Song, K. J., Chi, Z. P., Gao, L., Kang, G. H., \& Han, Y. Q. (2016). Weather Review of Shandong Province in Winter 2015 (December 2015-February 2016). Shandong Meteorology, 36, 55-57.

Sun, M., Meng, H., \& Fang, Y. (2015). A Typical Air Pollution Process in Qingdao City in Late Summer and Early Autumn of 2014. Earth, 1, 353-354.

Turner, D. B. (1964). A Diffusion Model for an Urban area. Journal of Applied Meteorology, 3, 83-91. https://doi.org/10.1175/1520-0450(1964)003<0083:ADMFAU>2.0.CO;2

Wan, M. B., Meng, X. X., Wang, Y. H., Kang, G. H., \& Li, J. (2015). Weather Review of Shandong Province in Winter 2014 (December 2014-February 2015). Shandong Meteorology, 35, 49-51.

Wang, B., \& Gao, H. W. (2008). Characteristics of Air Pollution Index in Coastal Cities of China. Ecology and Environmental Sciences, 17, 542-548. 
Wang, J. H., \& Guo, R. S. (1999). Study on the Method of Air Pollution Forecast in Qingdao. Journal of Qingdao University, 14, 60-62.

Wang, Y. Q., Zhang, X. Y., \& Draxler, R. R. (2009). TrajStat: GIS-Based Software That Uses Various Trajectory Statistical Analysis Methods to Identify Potential Sources from Long-Term Air Pollution Measurement Data. Environmental Modelling \& Software, 24, 938-939. https://doi.org/10.1016/j.envsoft.2009.01.004

Xu, H. X., Liu, R. H., Wang, Y., Zhang, Y. Y., Wang, M. Y., \& Zhang, Y. Q. (2017). Analysis of Air Quality Changes in Shandong Province from 2014 to 2016 and Sources of Pollutants from Polluted Weather in Typical Cities. China Population, Resources and Environment, 27, 132-135.

Xu, X. H., \& Yao, R. K. (1997). Study on the Contribution of Atmospheric Aerosol Ocean Factors in Qingdao Area. Marine Environmental Sciences, 16, 55-59.

You, H. C., Liu, W. D., \& Tan, J. R. (2010). Temporal Characteristics of Atmospheric Mixing Depth of Beijing. Meteorological Monthly, 36, 51-55.

Yuan, R. M., Ma, C. S., \& Fan, A. Y. (2013). Characteristics of the Capping Inversion above the Atmospheric Convective Boundary Layer. Journal of University of Science and Technology of China, 33, 247-252. 